\title{
Unification of Reaction Metrics for Green Chemistry: Applications to
} Reaction Analysis

John Andraos

Department of Chemistry, York University, Toronto, ON M3J 1P3 CANADA jandraos@yorku.ca

\section{Supplementary Material}

\section{Derivation of equation (7)}

$$
\begin{aligned}
E_{m} & =\frac{q y+w}{p y} \\
& =\frac{q y+a x+b x-q y-p y}{p y}, \text { since } w=a x+b x-q y-p y \\
& =\frac{a x+b x}{p y}-1 \\
& =\frac{a+b}{p \varepsilon}-1, \text { since } \varepsilon=\frac{y}{x} \\
& =\frac{1}{\varepsilon(A E)}-1, \text { since } A E=\frac{p}{a+b} \\
& =\frac{1}{R M E}-1, \text { since } R M E=\varepsilon(A E)
\end{aligned}
$$

\section{Derivation of equation (17)}

Given a general reaction

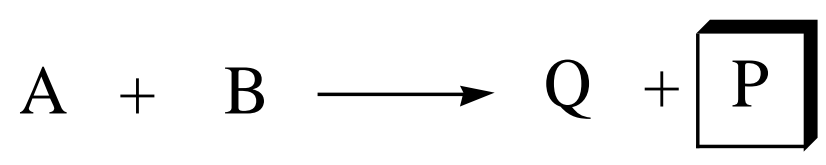

including masses of reaction solvent, $s$, catalyst, $c$, and post-reaction solvents and other materials, $\omega$, we have the law of conservation of mass given by 
$a x+b x+c+s+\omega=q y+p y^{\prime \prime}+w+\left(p y-p y^{\prime \prime}\right)+c+s+\omega$

where $x$ is the number of moles of reagents A and $\mathrm{B}, y$ is the number of moles of products $\mathrm{P}$ and $\mathrm{Q}$ formed in the reaction, $y^{\prime \prime}$ is the number of moles of product $\mathrm{P}$ collected after extraction and purification procedures, and $a, b, q$, and $p$ are the molecular weights of chemical species A, B, Q, and P. The parameter $w$ is the mass of unreacted starting materials.

Let $\varepsilon=\frac{y}{x}$ be the yield of reaction before work-up and and $\varepsilon^{\prime}=\frac{y^{\prime \prime}}{y}$ be the yield of collected product $\mathrm{P}$ after work-up and purification. Then, the overall yield of reaction product $\mathrm{P}$ after all operations is $\langle\varepsilon\rangle=\left(\frac{y}{x}\right)\left(\frac{y^{\prime \prime}}{y}\right)=\frac{y^{\prime \prime}}{x}$.

The environmental impact factor based on mass assuming that the waste is composed of mass of byproduct $\mathrm{Q}$, mass of unreacted starting materials, mass of product lost during the post-reaction operations, and masses of catalyst, reaction solvent, and all post-reaction materials used is given by

$$
E_{m}=\frac{q y+w+\left(p y-p y^{\prime \prime}\right)+c+s+\omega}{p y^{\prime \prime}}
$$

From the law of conservation of mass,

$$
w=a x+b x-q y-p y
$$

Substitution leads to

$$
\begin{aligned}
E_{m} & =\frac{q y+a x+b x-q y-p y+\left(p y-p y^{\prime \prime}\right)+c+s+\omega}{p y^{\prime \prime}}=\frac{a x+b x-p y^{\prime \prime}+c+s+\omega}{p y^{\prime \prime}} \\
& =\frac{a x+b x+c+s+\omega}{p y^{\prime \prime}}-1
\end{aligned}
$$


Since $\langle\varepsilon\rangle=\frac{y^{\prime \prime}}{x}$ we have

$$
E_{m}=\frac{a+b+\left(\frac{c+s+\omega}{x}\right)}{\left.p^{\prime} \varepsilon\right\rangle}-1
$$

Since the atom economy for the reaction is $A E=\frac{p}{a+b}$ then

$$
\begin{aligned}
& E_{m}=\frac{1+\left(\frac{c+s+\omega}{x}\right)\left(\frac{1}{a+b}\right)}{\left.A E_{\langle}^{\prime} \varepsilon\right\rangle}-1=\frac{1}{\frac{A E_{\langle}^{\prime} \varepsilon_{\rangle}}{1+\left(\frac{c+s+\omega}{x}\right)\left(\frac{1}{a+b}\right)}}-1 \\
& =\frac{1}{\frac{A E_{\langle\varepsilon\rangle}^{\prime} \varepsilon^{\prime}}{1+\left(\frac{c+s+\omega}{y^{\prime \prime}}\right)\left(\frac{\langle\varepsilon\rangle}{a+b}\right)}}-1=\frac{1}{\frac{\left.A E_{\langle}^{\prime} \varepsilon_{\rangle}\right\rangle}{1+\left(\frac{c+s+\omega}{y^{\prime \prime}}\right)\left(\frac{\langle\varepsilon\rangle}{p}\right)}}-1 \\
& =\frac{1}{\frac{\left.A E_{\langle}^{\prime} \varepsilon\right\rangle p y^{\prime \prime}}{p y^{\prime \prime}+(c+s+\omega)_{\langle} \varepsilon_{\rangle} A E}}-1
\end{aligned}
$$

By analogy with equation (7) the reaction mass efficiency is then

$$
R M E=\langle\varepsilon\rangle(A E)\left[\frac{p y^{\prime \prime}}{\left.p y^{\prime \prime}+{ }^{\prime} \varepsilon\right\rangle(A E)(c+s+\omega)}\right]=\frac{1}{1+E_{m}}
$$

\section{Derivation of equation (31)}

We start from the fundamental definition of environmental impact factor based on mass for the entire linear sequence 


$$
E_{\text {overall }}=\frac{\sum_{j=1}^{n} \bar{w}_{j}}{p_{n} x\left(\prod_{j=1}^{n} \varepsilon_{j}\right)}=\frac{\sum_{j=1}^{n}\left(w_{j}+q_{j} y_{j}+c_{j}+s_{j}+\omega_{j}\right)}{p_{n} x\left(\prod_{j=1}^{n} \varepsilon_{j}\right)},
$$

and for each step $j$ in the linear sequence we define the law of conservation of mass

$a_{j} x_{j}+b_{j} x_{j}+c_{j}+s_{j}+\omega_{j}=q_{j} y_{j}+p_{j} y_{j}+w_{j}+c_{j}+s_{j}+\omega_{j}$,

and the atom economy $(A E)_{j}=\frac{p_{j}}{a_{j}+b_{j}}$. The reaction yield for the first reaction

$\varepsilon_{1}=\frac{y_{1}}{x}\left(x_{1}=x\right)$ and that for subsequent reactions is $\varepsilon_{j}=\frac{y_{j}}{y_{j-1}}(\mathrm{j}=2,3, \ldots, \mathrm{n})$. All

variables are defined by analogy with the derivations of equations (7) and (17) and $n$ is the total number of steps. Then, the waste $w_{\mathrm{j}}$ is $w_{j}=a_{j} x_{j}+b_{j} x_{j}-q_{j} y_{j}-p_{j} y_{j}$ where $x_{1}=x, x_{2}=y_{1}, x_{2}=y_{3}$, etc. Substitution in $E_{\text {overall }}$ and following the same sequence of manipulations as for the derivations of equations (7) and (17) we have

$$
\begin{aligned}
& \frac{\sum_{j=1}\left(a_{j} x_{j}+b_{j} x_{j}-q_{j} y_{j}-p_{j} y_{j}+q_{j} y_{j}+c_{j}+s_{j}+\omega_{j}\right)}{p_{n} x\left(\prod_{j=1}^{n} \varepsilon_{j}\right)} \\
= & \frac{\sum_{j=1}^{n}\left(x_{j}\left(a_{j}+b_{j}\right)-p_{j} x_{j} \varepsilon_{j}+c_{j}+s_{j}+\omega_{j}\right)}{p_{n} x\left(\prod_{j=1}^{n} \varepsilon_{j}\right)}
\end{aligned}
$$




$$
=\frac{\sum_{j=1}^{n}\left(x_{j} p_{j}\left(\frac{1}{(A E)_{j}}-\varepsilon_{j}\right)+c_{j}+s_{j}+\omega_{j}\right)}{p_{n} x\left(\prod_{j=1}^{n} \varepsilon_{j}\right)}
$$

The first term in the sum is

$x_{1} p_{1}\left(\frac{1}{(A E)_{1}}-\varepsilon_{1}\right)+c_{1}+s_{1}+\omega_{1}=x_{1}\left(\frac{1}{(A E)_{1}}-\varepsilon_{1}\right)+c_{1}+s_{1}+\omega_{1}$

and the second term is

$x_{2} p_{2}\left(\frac{1}{(A E)_{2}}-\varepsilon_{2}\right)+c_{2}+s_{2}+\omega_{2}=y_{1} p_{2}\left(\frac{1}{(A E)_{2}}-\varepsilon_{2}\right)+c_{2}+s_{2}+\omega_{2}$

$=x \varepsilon_{1} p_{2}\left(\frac{1}{(A E)_{2}}-\varepsilon_{2}\right)+c_{2}+s_{2}+\omega_{2}$

The last term is

$x_{n} p_{n}\left(\frac{1}{(A E)_{n}}-\varepsilon_{n}\right)+c_{n}+s_{n}+\omega_{n}=y_{n-1} p_{n}\left(\frac{1}{(A E)_{n}}-\varepsilon_{n}\right)+c_{n}+s_{n}+\omega_{n}$

$=x \varepsilon_{1} \varepsilon_{2} \ldots \varepsilon_{n-1} p_{n}\left(\frac{1}{(A E)_{n}}-\varepsilon_{n}\right)+c_{n}+s_{n}+\omega_{n}$

Generally, for the $j$ th term $(j=2,3, \ldots, n)$

$x \varepsilon_{1} \varepsilon_{2} \ldots \varepsilon_{j-1} p_{j}\left(\frac{1}{(A E)_{j}}-\varepsilon_{j}\right)+c_{j}+s_{j}+\omega_{j}$

Dividing all terms by $p_{n} x\left(\prod_{j=1}^{n} \varepsilon_{j}\right)$ and summing yields 


$$
\begin{aligned}
& E_{\text {overall }}= \\
& \frac{1}{p_{n} x\left(\prod_{j=1}^{n} \varepsilon_{j}\right)} \sum_{j=1}^{n}\left(c_{j}+s_{j}+\omega_{j}\right)+ \\
& \frac{p_{1}}{p_{n}\left(\prod_{j=1}^{n} \varepsilon_{j}\right)}\left(\frac{1}{(A E)_{1}}-\varepsilon_{1}\right)+\frac{\varepsilon_{1} p_{2}}{p_{n}\left(\prod_{j=1}^{n} \varepsilon_{j}\right)}\left(\frac{1}{(A E)_{2}}-\varepsilon_{2}\right)+\frac{\varepsilon_{1} \varepsilon_{2} p_{3}}{p_{n}\left(\prod_{j=1}^{n} \varepsilon_{j}\right)}\left(\frac{1}{(A E)_{3}}-\varepsilon_{3}\right) \\
& +\ldots+\left(\frac{1}{\varepsilon_{n}}\right)\left(\frac{1}{(A E)_{n}}-\varepsilon_{n}\right) \\
& =\frac{1}{p_{n} x\left(\prod_{j=1}^{n} \varepsilon_{j}\right)} \sum_{j=1}^{n}\left(c_{j}+s_{j}+\omega_{j}\right)+ \\
& \frac{p_{1}}{p_{n}\left(\prod_{j=1}^{n} \varepsilon_{j}\right)}\left(\frac{1}{(A E)_{1}}-\varepsilon_{1}\right)+\frac{p_{2}}{p_{n}\left(\prod_{j=2}^{n} \varepsilon_{j}\right)}\left(\frac{1}{(A E)_{2}}-\varepsilon_{2}\right)+\frac{p_{3}}{p_{n}\left(\prod_{j=3}^{n} \varepsilon_{j}\right)}\left(\frac{1}{(A E)_{3}}-\varepsilon_{3}\right) \\
& +\ldots+\left(\frac{1}{\left.\varepsilon_{n}(A E)_{n}\right)}-1\right. \\
& \text { or } \\
& E_{\text {overall }}=\frac{1}{R M E_{\text {overall }}}-1 \\
& \text { the }
\end{aligned}
$$

where 
$\frac{1}{R M E_{\text {overall }}}=\frac{1}{p_{n} x\left(\prod_{j=1}^{n} \varepsilon_{j}\right)} \sum_{j=1}^{n}\left(c_{j}+s_{j}+\omega_{j}\right)+$

$\frac{p_{1}}{p_{n}\left(\prod_{j=1}^{n} \varepsilon_{j}\right)}\left(\frac{1}{(A E)_{1}}-\varepsilon_{1}\right)+\frac{p_{2}}{p_{n}\left(\prod_{j=2}^{n} \varepsilon_{j}\right)}\left(\frac{1}{(A E)_{2}}-\varepsilon_{2}\right)+\frac{p_{3}}{p_{n}\left(\prod_{j=3}^{n} \varepsilon_{j}\right)}\left(\frac{1}{(A E)_{3}}-\varepsilon_{3}\right)$

$+\ldots+\left(\frac{1}{\varepsilon_{n}(A E)_{n}}\right)$

\section{Woodward-Rabe Synthesis of Quinine}

Reaction 1

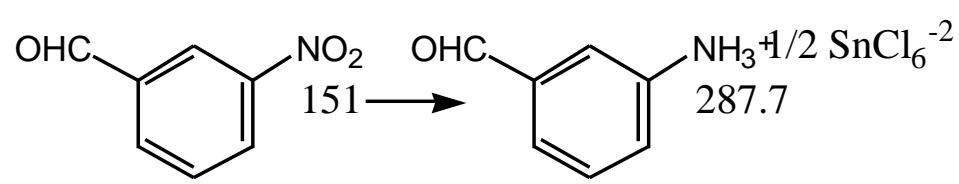

$\left\{\begin{array}{ll}3 \mathrm{SnCl}_{2} & 1006.2 \\ 12 \mathrm{HCl}\end{array} \quad\left\{\begin{array}{l}5 / 2 \mathrm{H}_{2} \mathrm{SnCl}_{6} \\ 2 \mathrm{H}_{2} \mathrm{O}\end{array}\right.\right.$

$\longrightarrow 2 \mathrm{NHCl}_{2}^{-2} \stackrel{\mathrm{OHC}}{\longrightarrow} \mathrm{NH}_{298.7}^{\mathrm{NH}_{3}+1 / 2 \mathrm{SnCl}_{6}^{-2}}$

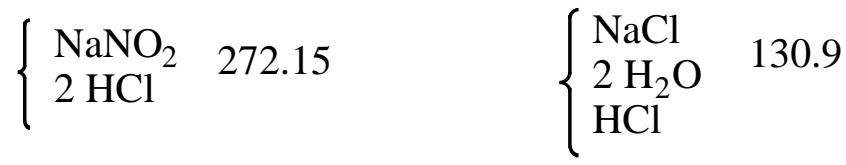<smiles>CCC(C=O)c1cccc(O)c1</smiles>

Reaction 2 


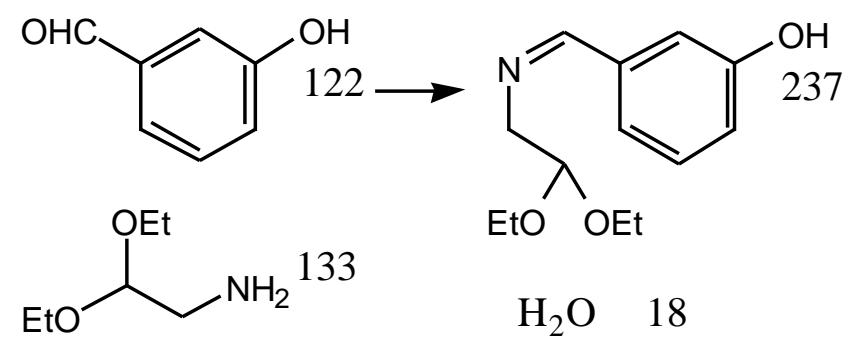

\section{$\underline{\text { Reaction } 3}$}<smiles>CCOC(C/N=C\c1cccc(O)c1)(OCC)OCC</smiles>

\section{$\underline{\text { Reaction } 4}$}

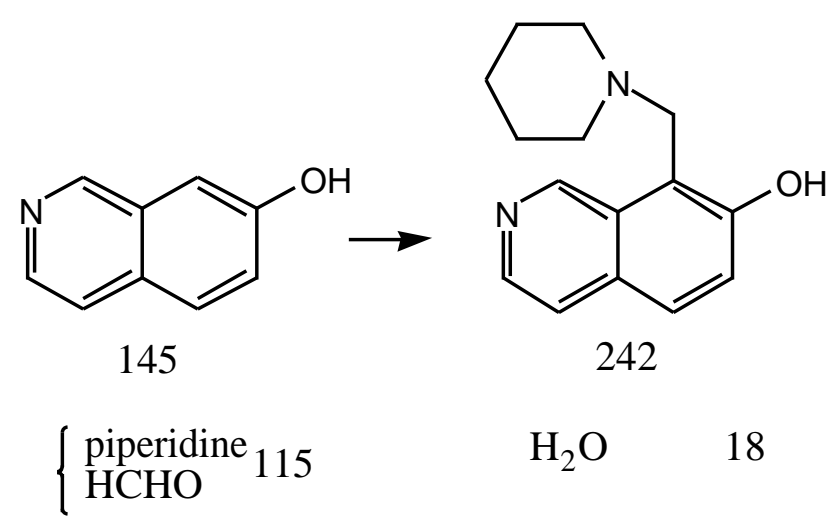

\section{$\underline{\text { Reaction } 5}$}

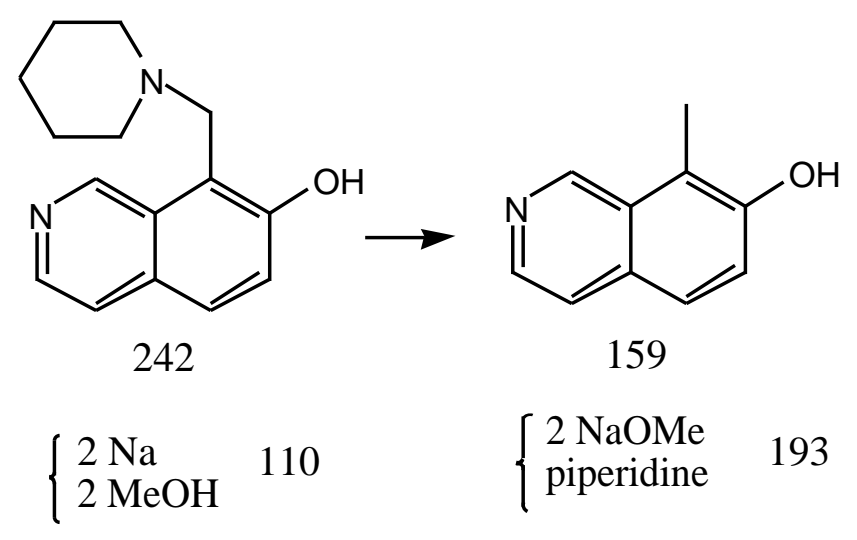


$\underline{\text { Reaction } 6}$<smiles>Cc1c(O)ccc2c1CNCC2</smiles>

\section{$\underline{\text { Reaction } 7}$}<smiles>Cc1c(O)ccc2c1CN(C(C)C)CC2</smiles>

$\underline{\text { Reaction } 8}$<smiles>Cc1c(O)ccc2c1CN(C(=O)C1CCCCC1)CC2</smiles>

$\underline{\text { Reaction } 9}$

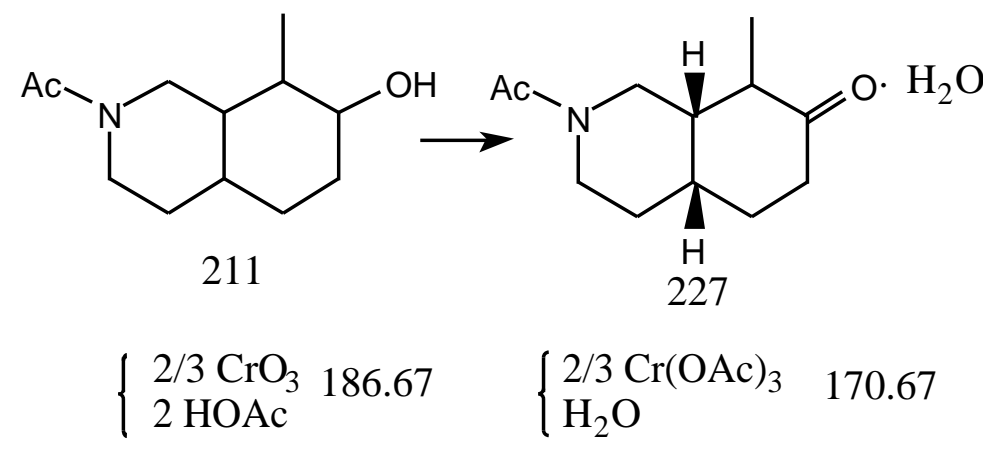

Reaction 10 
<smiles>CC1C(=O)CC[C@H]2CCN(C3CCCC3)C[C@@H]12</smiles>

227<smiles>CCN1CC[C@H]2CCC(=O)C(C)[C@H]2C1</smiles>

209<smiles>CC1C(=O)CC[C@H]2CCN(C(=O)Cl)C[C@@H]12</smiles>

209

$\left\{\begin{array}{l}\mathrm{Na} \\ \mathrm{EtOH} \\ \mathrm{EtONO}\end{array}\right.$<smiles></smiles>

284

$\underline{\text { Reaction } 11}$<smiles>CCOC(=O)CC[C@]1([18OH])CCN(C(=O)OCC)C[C@@H]1C(C)=N</smiles>

$\mathrm{H}_{2} \quad 2$

Reaction 12<smiles>[2H][C@]1(CCC(=O)OCC)CCN(C(=O)OCC)C[C@H]1C(C)N</smiles>

\section{$\underline{\text { Reaction } 13}$}


<smiles>CCOCC[C@H]1CCN(C)C[C@H]1C(C)N(C)[I-]I</smiles>

440

$2 \mathrm{NaOH} \quad 80$<smiles>C=C[C@H]1CN(C)CC[C@@H]1CCC(=O)O</smiles>

225<smiles>C=C[C@H]1CN(C(=O)O)CC[C@@H]1CCC(=O)O</smiles>

225
$\left\{\begin{array}{l}\mathrm{H}_{2} \mathrm{O} \\ \mathrm{NMe}_{3} \quad 295 \\ \mathrm{NaI} \\ \mathrm{NaOEt}\end{array}\right.$<smiles>C=C[C@H]1CN(C(N)=O)CC[C@H]1CCC(=O)O</smiles>

226

\section{Reaction 14}<smiles>C=C[C@H]1CN(C(N)=O)CC[C@H]1CCC(=O)O</smiles>

226

$\mathrm{HCl} \quad 36.45$<smiles>C=C[C@H]1CN(C(N)=O)CC[C@@H]1CCC(=O)O</smiles>

262.45<smiles>C=C[C@H]1CN(C(=O)c2ccccc2)CC[C@H]1CCC(=O)OCC</smiles>

315

$\left\{\begin{array}{l}2 \mathrm{EtOH} \\ \mathrm{PhCOCl}\end{array}\right.$

232.45

$\begin{cases}\mathrm{HCl} & \\ \mathrm{H}_{2} \mathrm{NCOOEt} \mathrm{HCl} & 179.9 \\ \mathrm{H}_{2} \mathrm{O} & \end{cases}$ 
$\underline{\text { Reaction } 15}$<smiles>C=C[C@H]1CN(C(=O)c2ccccc2)CC[C@H]1CCC(=O)OCC</smiles>

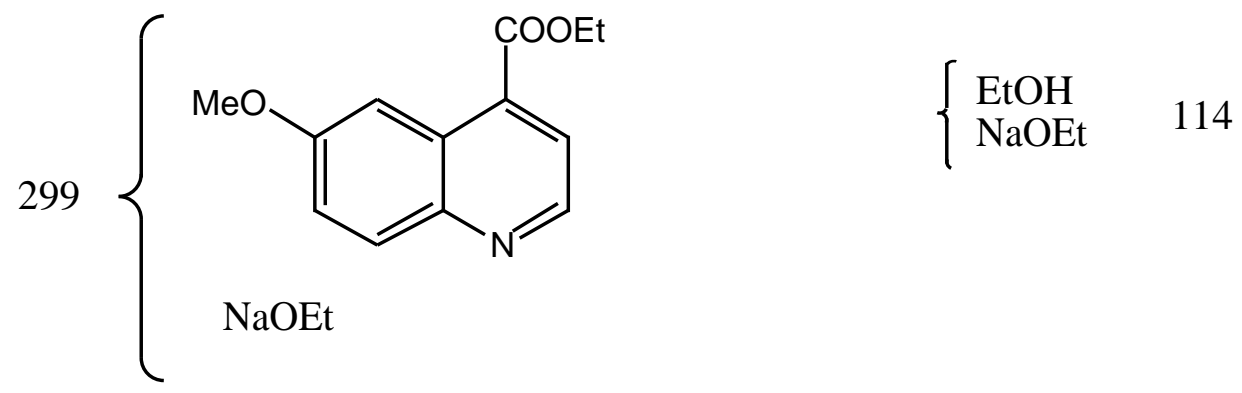

$\underline{\text { Reaction } 16}$<smiles>C=C[C@H]1CN(C(=O)c2ccccc2)CC[C@@H]1CC(C(=O)Br)C(=O)OCC</smiles><smiles>C=C[C@H]1CN([TlH]C)CC[C@H]1CCC(=O)Br</smiles>

$2 \mathrm{H}_{2} \mathrm{O} \quad 36$

$\left\{\begin{array}{l}\mathrm{EtOH} \\ \mathrm{CO}_{2} 212 \\ \mathrm{PhCOOH}\end{array}\right.$

$\underline{\text { Reaction } 17}$<smiles>C=C[C@H]1CNCC[C@@H]1CCC(=O)Br</smiles>

$\left\{\begin{array}{l}2 \mathrm{NaOH} \\ \mathrm{Br}_{2}\end{array}\right.$<smiles>C=C[C@H]1CN(Br)CC[C@@H]1CCC(=O)Br</smiles>

$\begin{cases}\mathrm{NaBr} & \\ \mathrm{H}_{2} \mathrm{O} & 160.9 \\ \mathrm{NaOH} & \end{cases}$

\section{$\underline{\text { Reaction } 18}$}




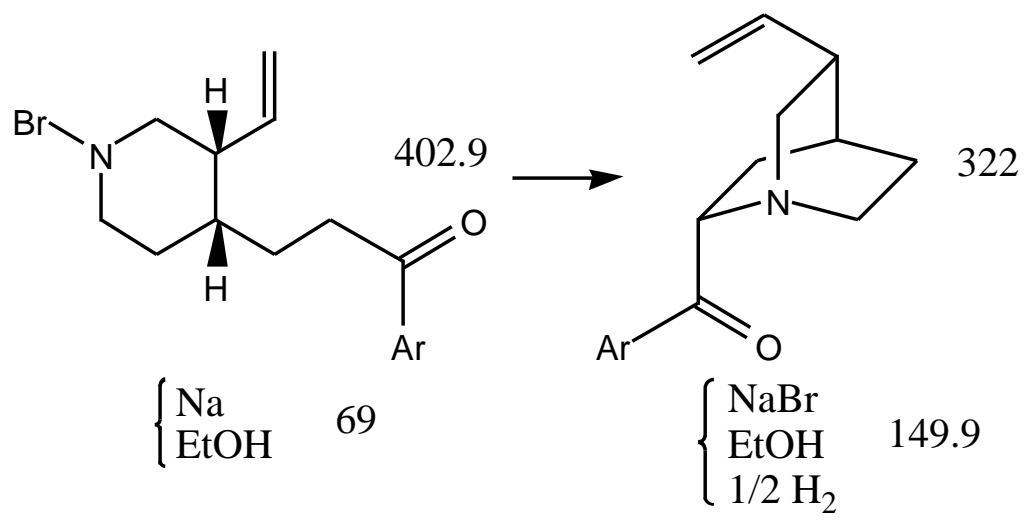

$\underline{\text { Reaction } 19}$

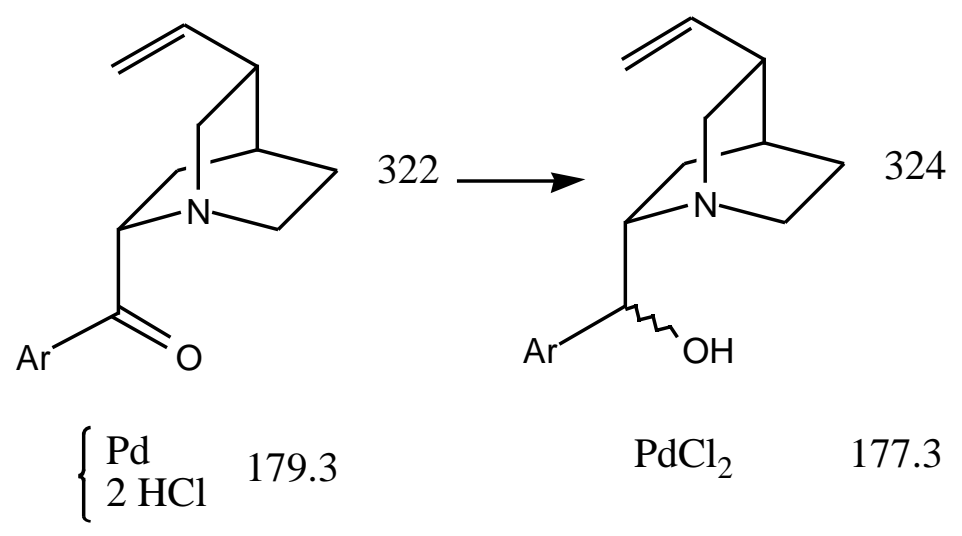

\section{Stork Synthesis of Quinine}

\section{$\underline{\text { Reaction } 1}$}

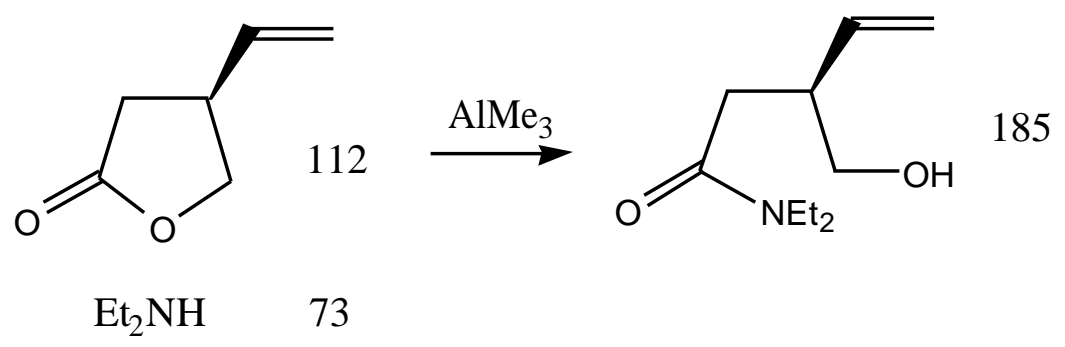

$\underline{\text { Reaction } 2}$ 


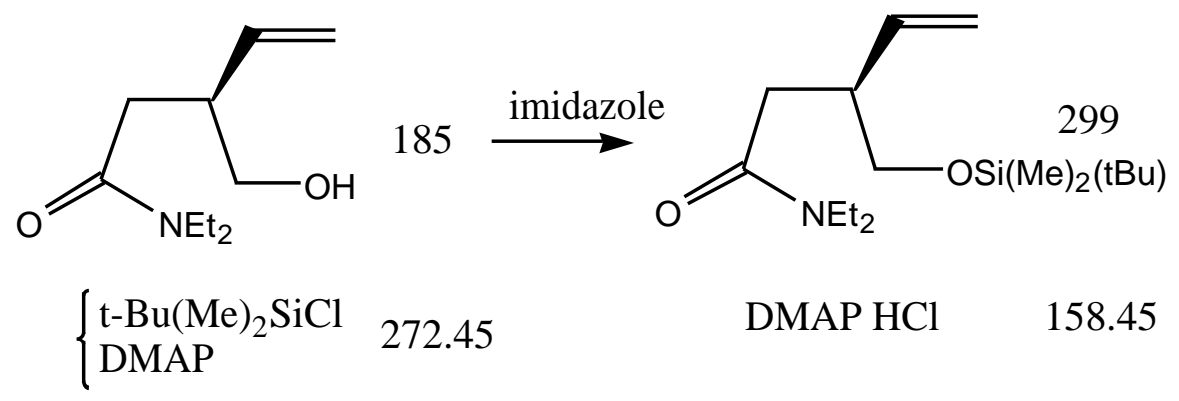

\section{$\underline{\text { Reaction } 3}$}

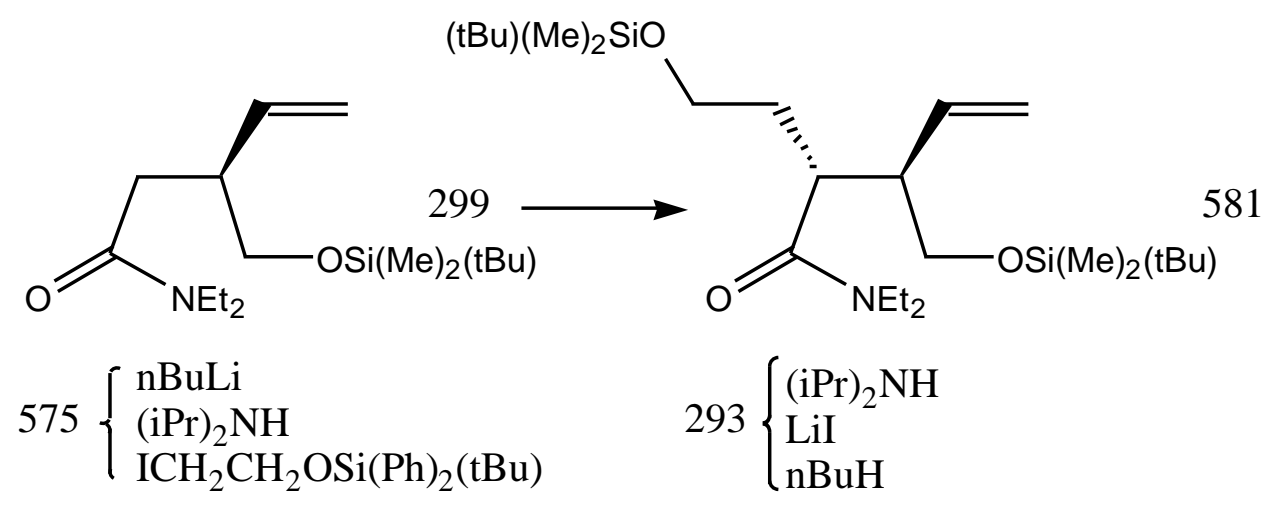

$\underline{\text { Reaction } 4}$

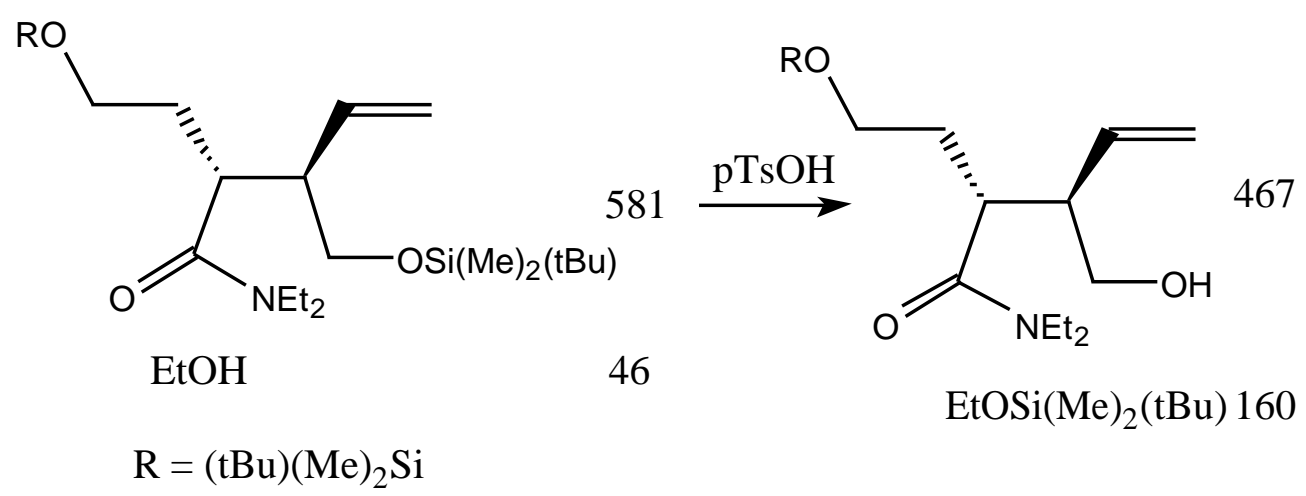

\section{$\underline{\text { Reaction } 5}$}




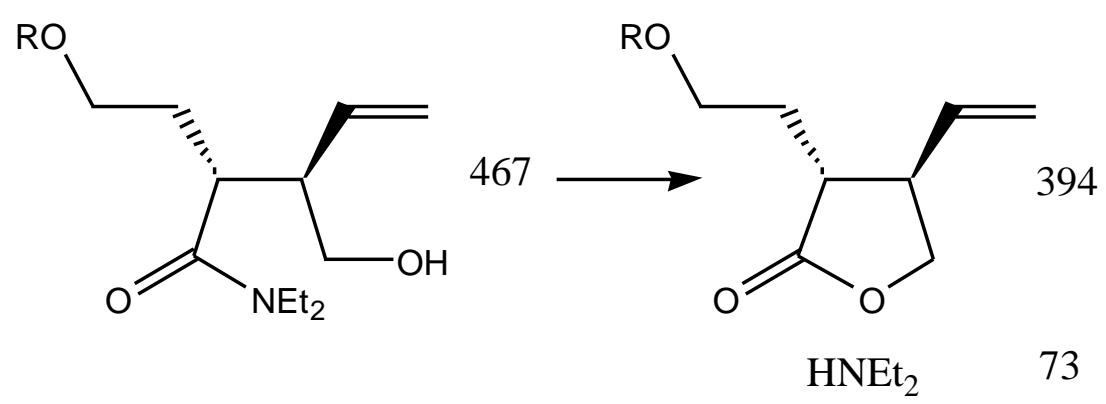

$\underline{\text { Reaction } 6}$

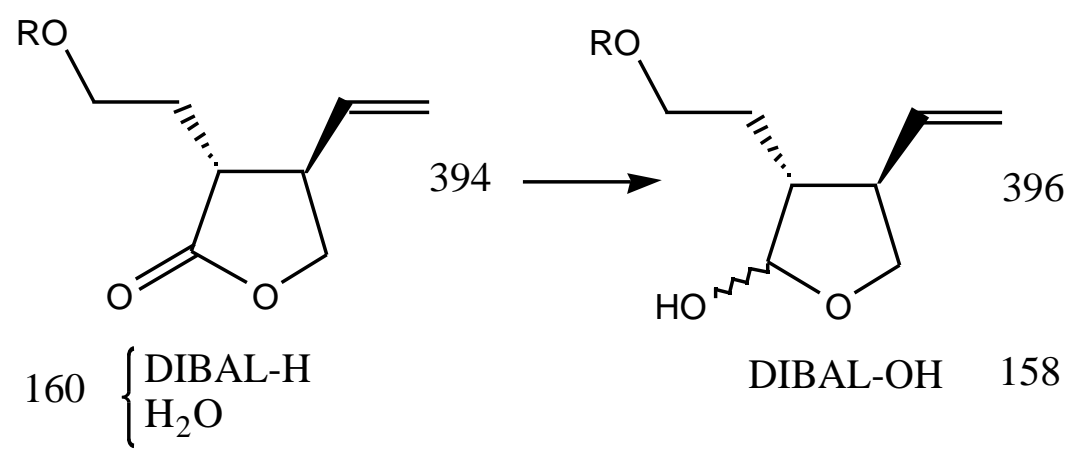

$\underline{\text { Reaction } 7}$

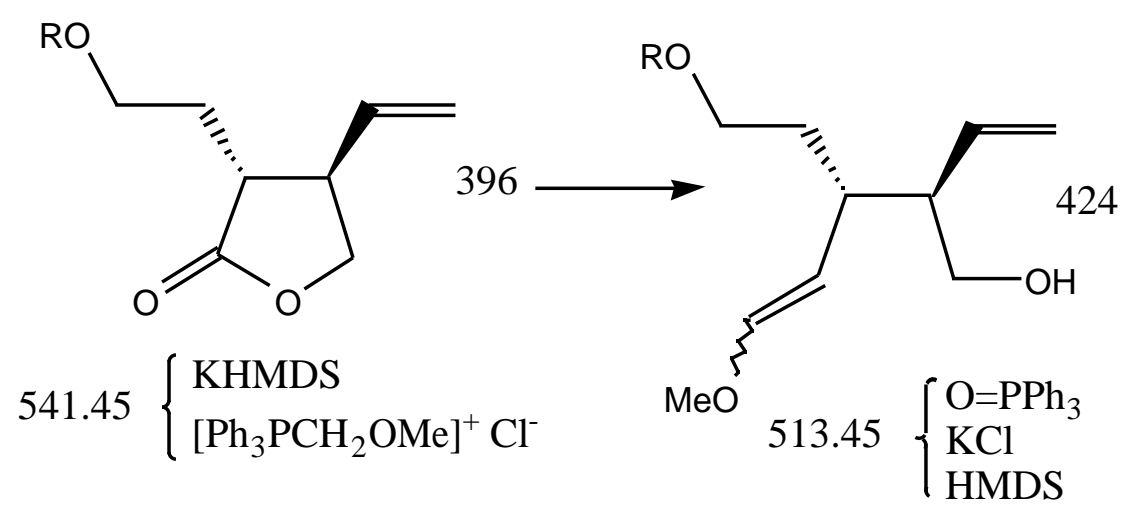

$\underline{\text { Reaction } 8}$ 


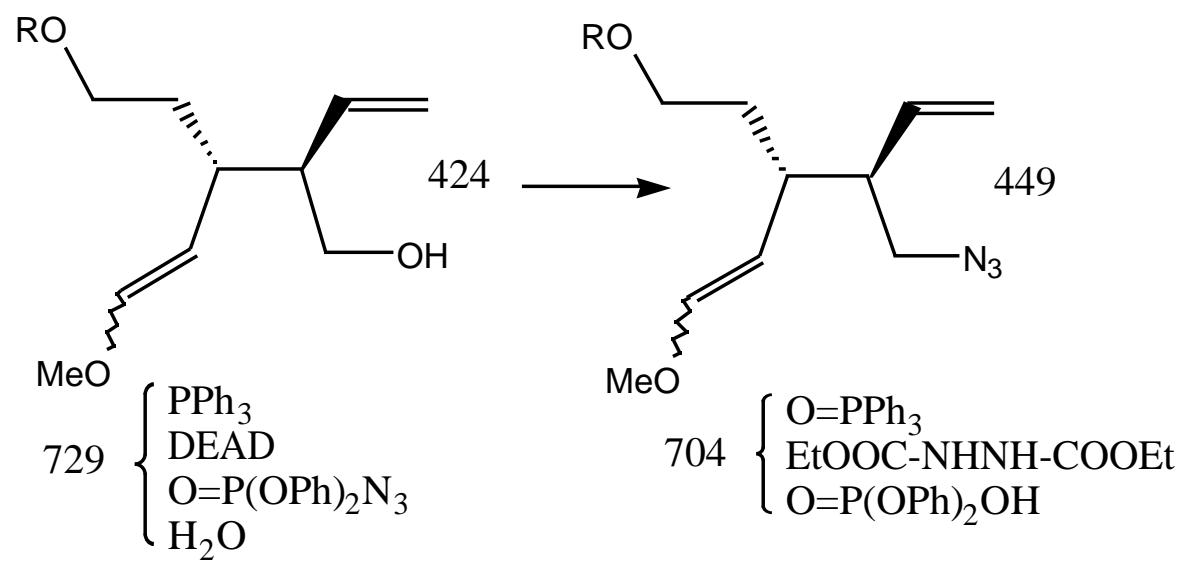

$\underline{\text { Reaction } 9}$

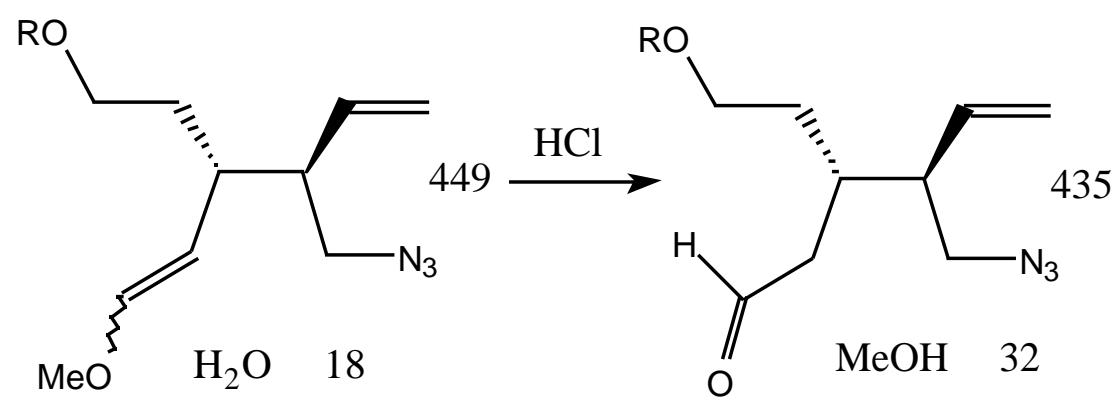

Reaction 10

173<smiles>COc1ccc2ncc(C)cc2c1</smiles>

107

LDA

$(\mathrm{iPr})_{2} \mathrm{NH}$

101 


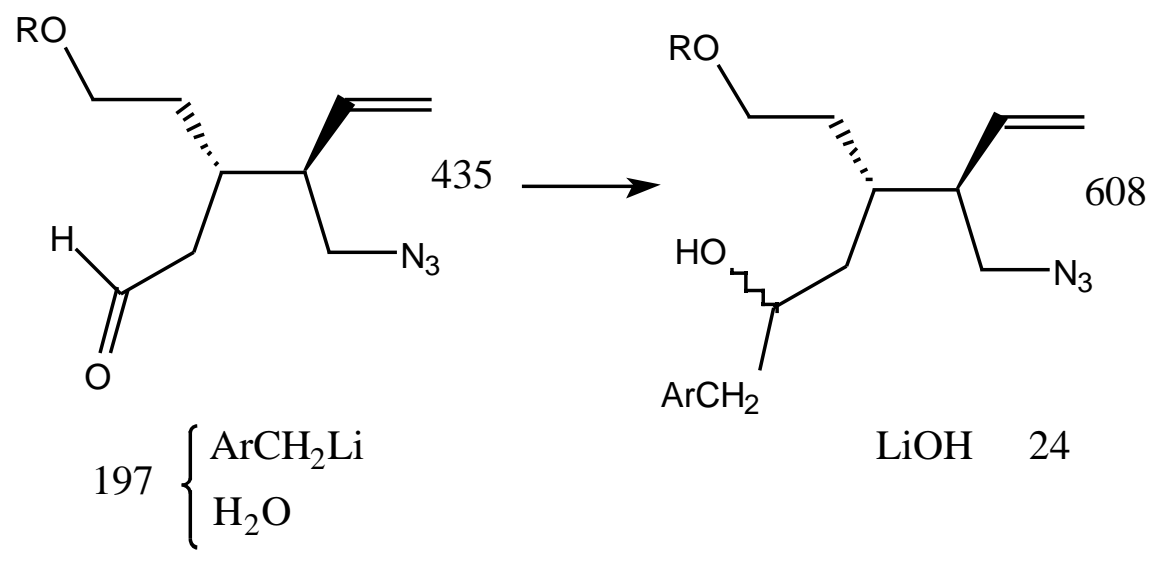

\section{$\underline{\text { Reaction } 11}$}

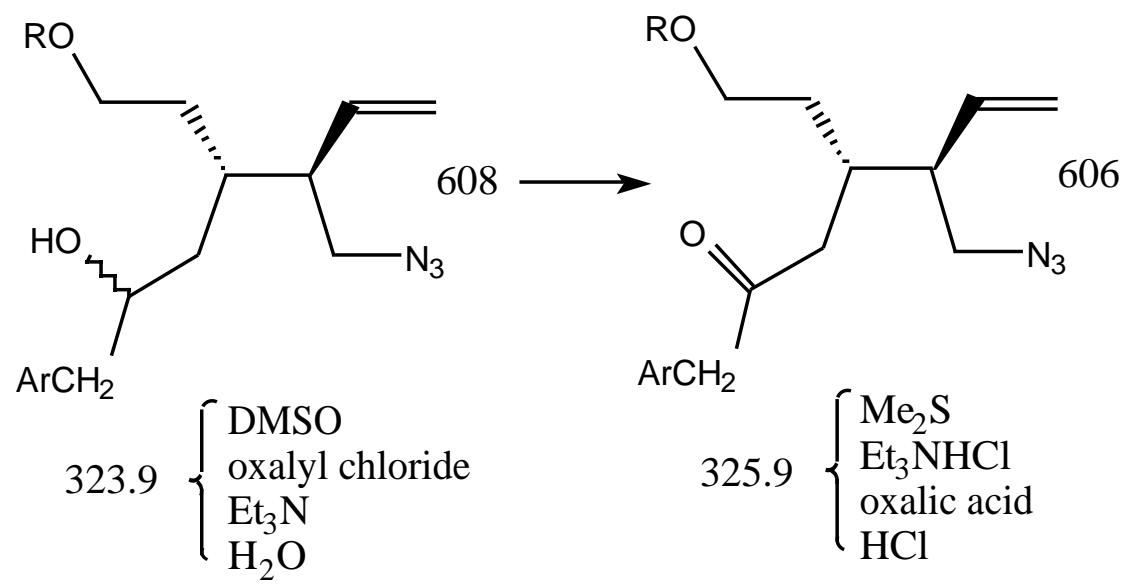

\section{Reaction 12}

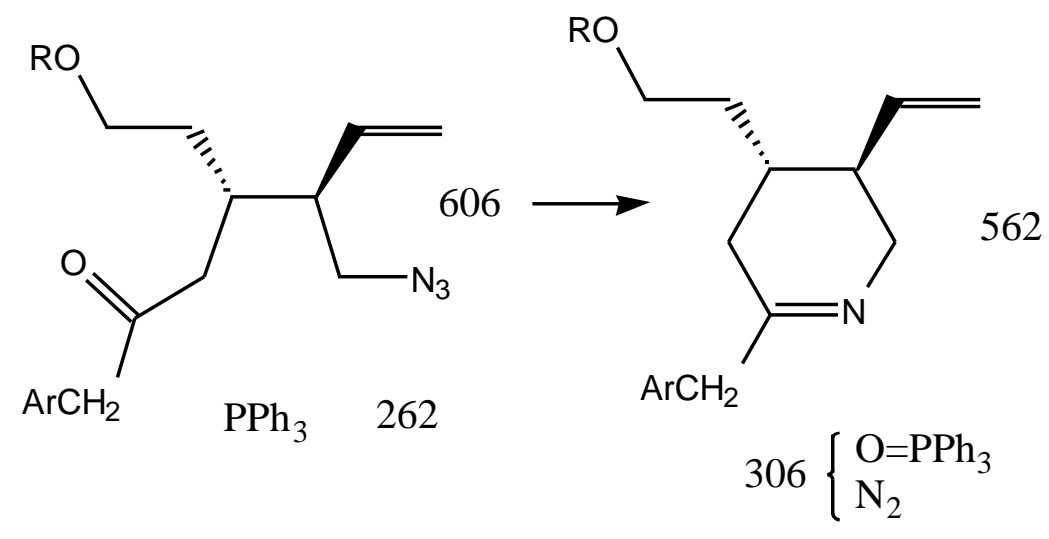

$\underline{\text { Reaction } 13}$ 

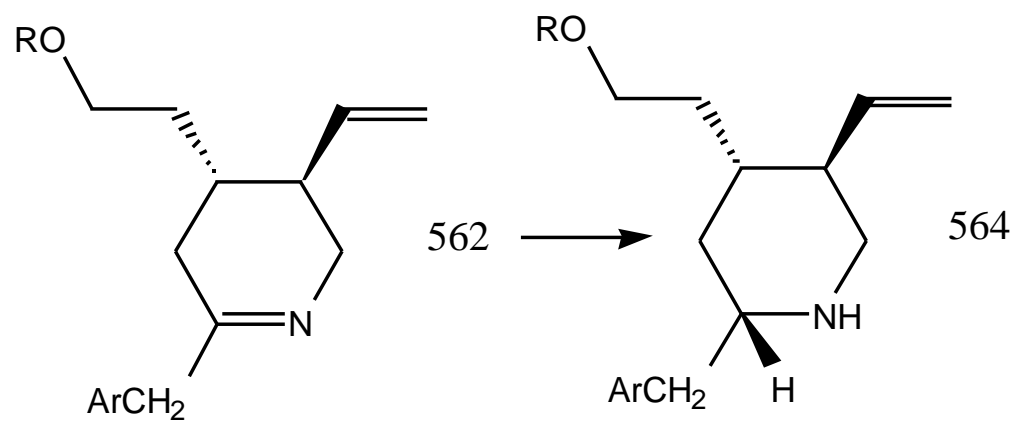

$27.5\left\{\begin{array}{l}1 / 4 \mathrm{NaBH}_{4} \\ \mathrm{H}_{2} \mathrm{O}\end{array}\right.$

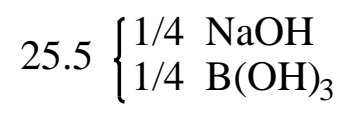

\section{$\underline{\text { Reaction } 14}$}
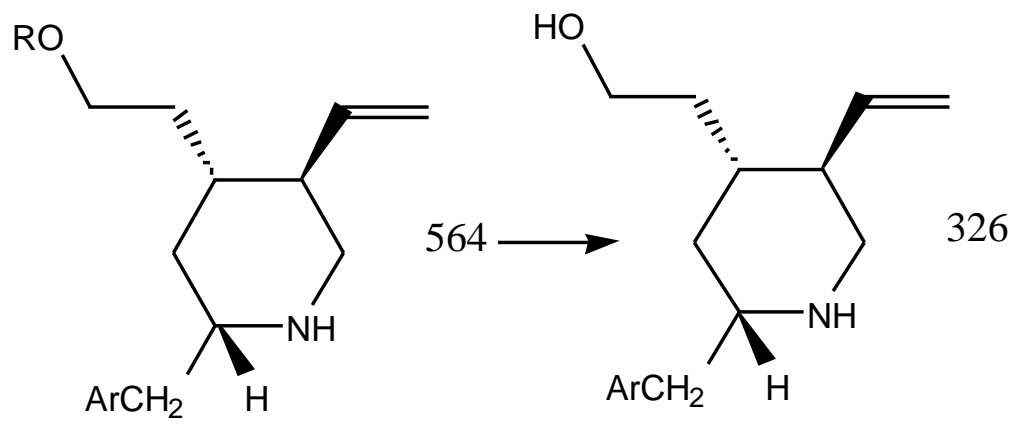

HF 20

FR 258

Reaction 15
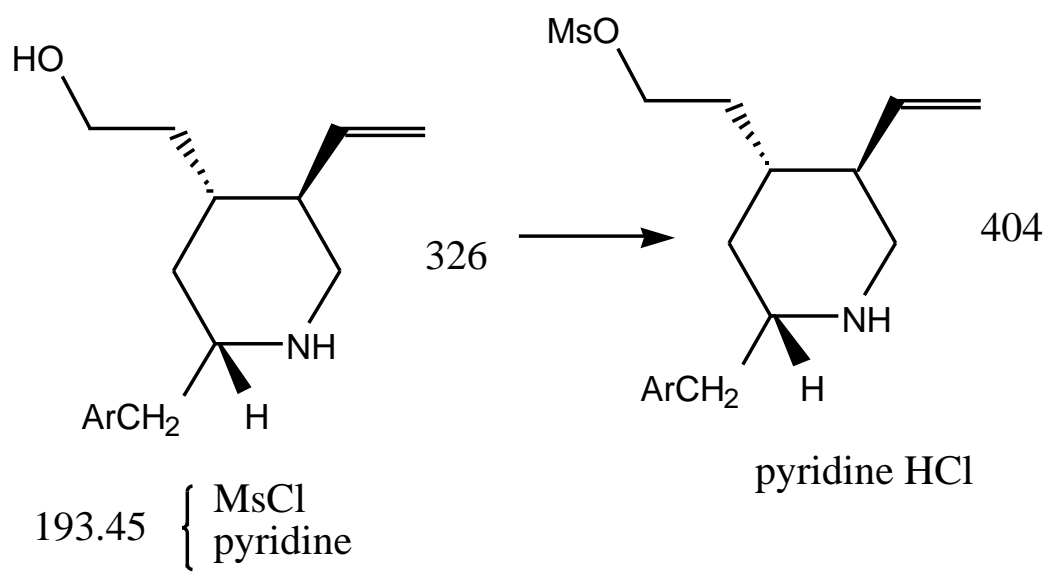

pyridine $\mathrm{HCl} \quad 115.45$

$\underline{\text { Reaction } 16}$ 


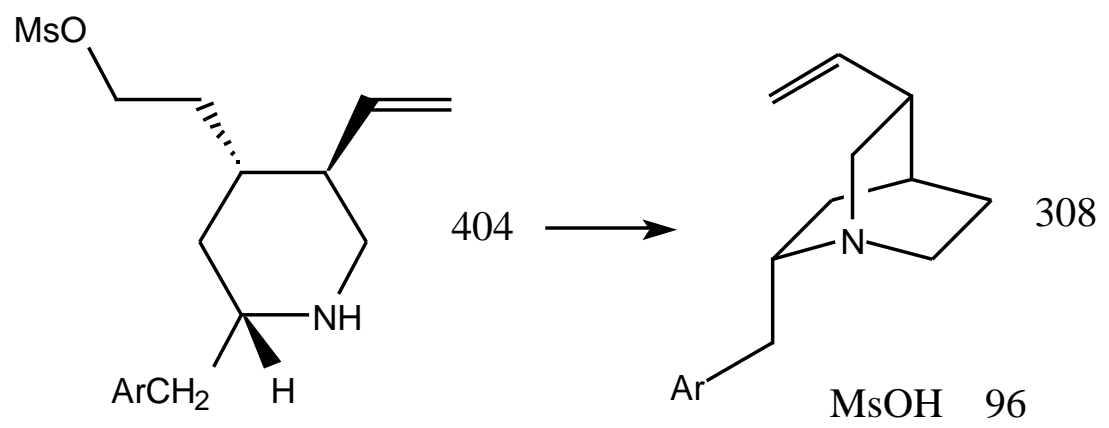

\section{$\underline{\text { Reaction } 17}$}

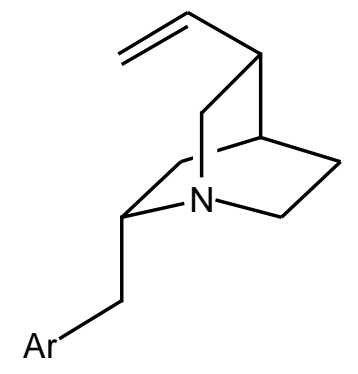

$98\left\{\begin{array}{l}2 \mathrm{NaH} \\ \mathrm{O}_{2} \\ \mathrm{H}_{2} \mathrm{O}\end{array}\right.$

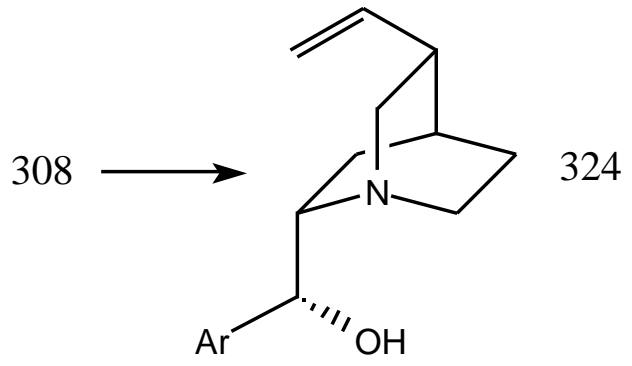

$82\left\{\begin{array}{l}\mathrm{H}_{2} \\ 2 \mathrm{NaOH}\end{array}\right.$

\section{Derivation of Equation (44)}

After cycle 1 the RME with respect to the desired (-) alcohol product is

$$
R M E_{1}^{(-)}=\frac{172 x\left(1-\lambda_{1}\right) \lambda_{2}}{x\left[16+27.5 \lambda_{1}+172\right]+\omega}
$$

In cycle 2 we have for the oxidation step: 


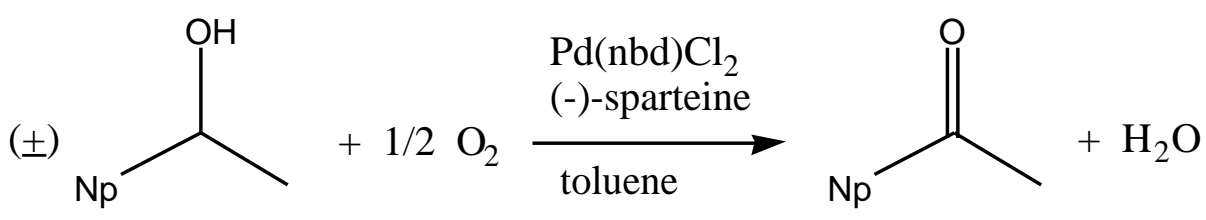

MW

172

32

170

18

moles

$\mathrm{x} \lambda_{1} \quad 0.5 \times \lambda_{1}$

$\mathrm{x}\left(\lambda_{1}\right)^{2} \quad \mathrm{x}\left(\lambda_{1}\right)^{2}$

mass (g)

$172 \times \lambda_{1} \quad 16 \times \lambda_{1}$

$170 \times\left(\lambda_{1}\right)^{2} \quad 18 \times\left(\lambda_{1}\right)^{2}$

and for the reduction step:

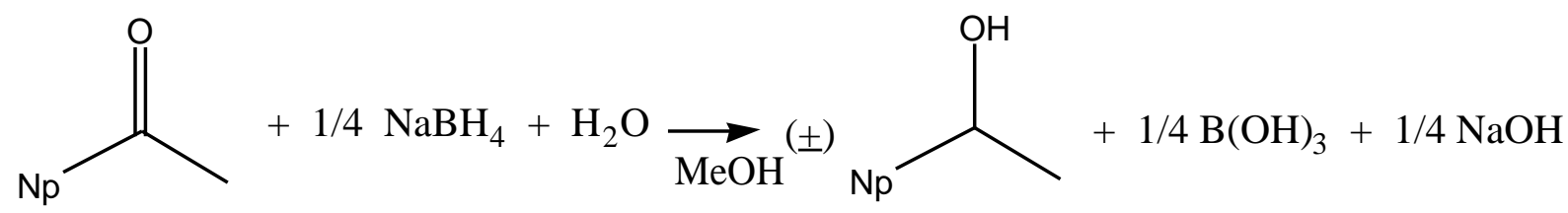

MW

170

38

18

172

62

40

moles

$$
\mathrm{x}\left(\lambda_{1}\right)^{2}
$$

$0.25 \mathrm{x}\left(\lambda_{1}\right)^{2} \quad \mathrm{x}\left(\lambda_{1}\right)^{2}$

$x\left(\lambda_{1}\right)^{2}$

$0.25 \times\left(\lambda_{1}\right)^{2}$

$0.25 \times\left(\lambda_{1}\right)^{2}$

mass (g) $\quad 170 \times\left(\lambda_{1}\right)^{2} \quad 9.5 \times\left(\lambda_{1}\right)^{2} \quad 18 \times\left(\lambda_{1}\right)^{2}$

$172 \times\left(\lambda_{1}\right)^{2}$

$15.5 \times\left(\lambda_{1}\right)^{2} \quad 10 \times\left(\lambda_{1}\right)^{2}$

After cycle 2 the cumulative RME with respect to the (-) alcohol is

$$
\begin{aligned}
R M E_{2}^{(-)} & =\frac{172 x\left(1-\lambda_{1}\right) \lambda_{2}+172 x \lambda_{1}\left(1-\lambda_{1}\right) \lambda_{2}}{x\left[16+27.5 \lambda_{1}+172\right]+x\left[16 \lambda_{1}+27.5 \lambda_{1}^{2}\right]+2 \omega} \\
& =\frac{172 x\left(1-\lambda_{1}\right)\left(1+\lambda_{1}\right) \lambda_{2}}{x\left[16\left(1+\lambda_{1}\right)+27.5 \lambda_{1}\left(1+\lambda_{1}\right)+172\right]+2 \omega} \\
& =\frac{172 x\left(1-\lambda_{1}\right)\left(1+\lambda_{1}\right) \lambda_{2}}{x\left[\left(16+27.5 \lambda_{1}\right)\left(1+\lambda_{1}\right)+172\right]+2 \omega}
\end{aligned}
$$

Similarly, after cycle 3 
$R M E_{3}^{(-)}=\frac{172 x\left(1-\lambda_{1}\right)\left(1+\lambda_{1}+\lambda_{1}^{2}\right) \lambda_{2}}{x\left[\left(16+27.5 \lambda_{1}\right)\left(1+\lambda_{1}+\lambda_{1}^{2}\right)+172\right]+3 \omega}$

Generally, after cycle $m$

$$
R M E_{m}^{(-)}=\frac{172 x\left(1-\lambda_{1}\right)\left(1+\lambda_{1}+\lambda_{1}^{2}+\ldots \lambda_{1}{ }^{m-1}\right) \lambda_{2}}{x\left[\left(16+27.5 \lambda_{1}\right)\left(1+\lambda_{1}+\lambda_{1}^{2}+\ldots \lambda_{1}{ }^{m-1}\right)+172\right]+m \omega}
$$

Now,

$1+\lambda_{1}+\lambda_{1}{ }^{2}+\ldots \lambda_{1}{ }^{m-1}=\frac{1-\lambda_{1}{ }^{m}}{1-\lambda_{1}}$ for $0<\lambda_{1}<1$

Therefore,

$$
\begin{aligned}
R M E_{m}^{(-)} & =\frac{172 x\left(1-\lambda_{1}\right)\left(\frac{1-\lambda_{1}{ }^{m}}{1-\lambda_{1}}\right) \lambda_{2}}{x\left[\left(16+27.5 \lambda_{1}\right)\left(\frac{1-\lambda_{1}^{m}}{1-\lambda_{1}}\right)+172\right]+m \omega} \\
& =\frac{172 x\left(1-\lambda_{1}\right)\left(1-\lambda_{1}{ }^{m}\right) \lambda_{2}}{x\left[\left(16+27.5 \lambda_{1}\right)\left(1-\lambda_{1}^{m}\right)+172\left(1-\lambda_{1}\right)\right]+m \omega\left(1-\lambda_{1}\right)} \\
R M E_{m}^{(-)} & =\frac{172 x\left(1-\lambda_{1}\right)\left(1-\lambda_{1}^{m}\right) \lambda_{2}}{x\left[\left(16+27.5 \lambda_{1}\right)\left(1-\lambda_{1}^{m}\right)+172\left(1-\lambda_{1}\right)\right]+m\left(1-\lambda_{1}\right) \omega}
\end{aligned}
$$

which is equation (44). 